\title{
HIGH-RESOLUTION SPECTROSCOPY OF BETA CEPHEI
}

\section{STARS}

\author{
C. WAELKENS, H. VAN WINCKEL and K. DE MEY \\ Instituut voor Sterrenkunde \\ Celestijnenlaan 200B, B-3001 Leuven (Belgium)
}

\begin{abstract}
.
We give a progress report on an observational program intended to determine detailed chemical abundances of $\beta$ Cephei stars and constant stars with similar temperature and gravity. There is some evidence that non-variable stars have a lower metal content than variables, as the recently found pulsation mechanism would suggest.
\end{abstract}

\section{Introduction}

The long-lasting enigma of the pulsation mechanism of the $\beta$ Cephei stars now seems to be solved, with the inclusion of the new opacities in the theoretical models (Dziembowski, this conference). An interesting corollary of the metal-opacity induced instability is that, since a three-fold increase of the metal opacity sets a limit from stable to unstable models, then the metal abundance is a critical parameter for the behavior of a star in the $\beta$ Cephei instability strip. For instance, the $\beta$ Cephei phenomenon should be absent in the Magellanic Clouds, as indeed seems to be corroborated by observation (Sterken and Jerzykiewicz 1988, Balona 1993).

Observational tests for the mechanism can also be devised in our Galaxy. Is the difference between constant and pulsating stars one of metal abundance? Is there a relation between metal abundance and pulsation amplitude? In order to address these questions, we have undertaken a highresolution spectroscopic study of galactic $\beta$ Cephei stars and of constant stars with similar photometric colors. We present here a first report on this project.

\section{Observations}

High-resolution spectra for northern $\beta$ Cephei stars have been obtained with the Aurélie spectrograph at the $1.5 \mathrm{~m}$ Telescope at Haute-Provence Observatory in France, during October 1992. High-resolution spectra for southern $\beta$ Cephei stars have been obtained with the CES spectrograph at the $1.4 \mathrm{~m}$ CAT Telescope at ESO, Chile, during April 1992 and January and February 1993.

Optical photometric parameters, such as the X-index of the Geneva Photometric System, are sensitive temperature estimators in the range defined by the $\beta$ Cephei stars. A fortunate circumstance is also that the instability 
strip occurs in the temperature range where lines from three different ionizations stages of silicon can be observed (Si II-IV), so that also accurate spectroscopic estimates of the effective temperature are possible. On the other hand, optical photometric colors are only slightly sensitive to gravity in this spectral range.

It is somewhat unfortunate that iron lines are scarce in optical spectra of early-B-type stars. The best line seems to be the FeIII line at $4165 \AA$ which is unblended and occurs in the linear part of the curve of growth.

\section{First Results and Prospects}

The observational temperature parameters (Geneva X index, uvby photometry, Si-line ratios) correlate well with directly derived effective temperatures and with effective temperatures derived from model atmospheres. However, temperatures derived from published non-LTE analyses appear to be systematically too high when compared with those obtained with direct methods.

When the equivalent width of the FeIII-4165 line is plotted as a function of effective temperature, it appears that on average the equivalent width is smaller for non-variable stars than for the $\beta$ Cephei stars. The mean effect amounts to some $30 \%$, and individual exceptions occur. The large-amplitude variables are not characterized by unusually strong iron abundances.

The next step in the investigation will be a quantitative analysis of the abundances of the metals.

\section{The References}

Balona, L.A., 1993, Monthly Notices of the RAS256, 425

Sterken, C., Jerzykiewicz, M., 1988, Monthly Notices of the RAS235, 565 